Bull. Austral. Math. Soc.

VOL. 56 (1997) $[81-86]$

\title{
RATIONAL INTERPOLATION TO $|x|$ AT THE CHEBYSHEV NODES
}

\author{
Lev Brutman and Eli Passow
}

\begin{abstract}
Recently the authors considered Newman-type rational interpolation to $|x|$ induced by arbitrary sets of interpolation nodes and showed that under mild restrictions on the location of the interpolation nodes, the corresponding sequence of rational interpolants converges to $|x|$. In the present paper we consider the special case of the Chebyshev nodes which are known to be very efficient for polynomial interpolation. It is shown that, in contrast to the polynomial case, the approximation of $|x|$ induced by rational interpolation at the Chebyshev nodes has the same order as rational interpolation at equidistant points.
\end{abstract}

\section{INTRODUCTION}

The function $|x|$ has been the focus of much research in approximation theory over the years. Its fundamental role in polynomial approximation is well illustrated by Lebesgue's proof of the Weierstrass approximation theorem, which is based solely on the fact that the single function $|x|$ can be approximated. However, as was shown by Bernstein $[\mathbf{1}]$, the order of the best uniform approximation of $|x|$ by polynomials is only $O\left(n^{-1}\right)$.

In contrast to this, Newman [5] demonstrated that rational approximation to $|x|$ is much more favorable, namely $|x|$ may be approximated uniformly by rational functions at an exponential rate. Newman's result generated a great deal of research, much of which focused on the problem of sharpening the asymptotic results for the error in the best rational approximation. The most recent result in this direction is the proof of the so-called " 8 " conjecture by Stahl. (See [7], where the main result is presented and an extensive historical review is given).

In a recent paper [2] the authors considered Newman-type rational approximation induced by arbitrary sets of interpolation points. Let $X=\left\{0<x_{1}^{(n)}<x_{2}^{(n)}<\ldots<\right.$ $\left.x_{n}^{(n)} \leqslant 1\right\}$ be a set of $n$ distinct points in $(0,1]$ and let $p(x)=\prod_{k=1}^{n}\left(x+x_{k}^{(n)}\right)$. (In the sequel, when there is no possibility for confusion, the superscript $(n)$ will be omitted.) The rational function, corresponding to the set $X$, is defined by

$$
r_{n}(X ; x)=x \frac{p(x)-p(-x)}{p(x)+p(-x)}
$$

Received 28th August, 1996

Copyright Clearance Centre, Inc. Serial-fee code: 0004-9729/97 \$A2.00+0.00. 
It can be easily verified that $r_{n}(X ; x)$ interpolates $|x|$ at the following set of $2 n+1$ points: $\left\{-x_{n}, \ldots,-x_{1}, 0, x_{1}, \ldots, x_{n}\right\}$. Since $r_{n}(X ; x)$ as well as $|x|$ are even functions, the study of the approximation error $e_{n}(X ; x)=|x|-r_{n}(X ; x)$ may be restricted to the interval $[0,1]$, where it can be represented in the following form:

where

$$
\begin{aligned}
& e_{n}(X ; x)=\frac{2 x h_{n}(X ; x)}{1+h_{n}(X ; x)}, \quad 0 \leqslant x \leqslant 1, \\
& h_{n}(X ; x)=\frac{p(-x)}{p(x)}=\prod_{k=1}^{n} \frac{x_{k}-x}{x_{k}+x} .
\end{aligned}
$$

In the sequel we shall use the following general estimates which were proved in $[2]$ :

StATEMENT 1.1. Let $S_{1}=S_{1}^{(n)}(X)=\sum_{k=1}^{n} x_{k}^{(n)}$. Then

$$
\begin{aligned}
& \left|h_{n}(X ; x)\right| \leqslant e^{-x S_{1}}, \quad 0 \leqslant x \leqslant 1, \\
& \left|e_{n}(X ; x)\right| \leqslant \frac{2}{S_{1}}, \quad-1 \leqslant x \leqslant 1 .
\end{aligned}
$$

Statement 1.2 . Let $A_{n}=A_{n}(X)=1 / \sum_{k=1}^{n} x_{k}{ }^{-1}$. Then

$$
\left|e_{n}(X ; x)\right| \leqslant 1 / A_{n}, \quad x \in\left[-x_{1}, x_{1}\right] .
$$

Note that (3) implies in particular that for the set $E$ of equally spaced points

$$
\left|e_{n}(E ; x)\right| \leqslant \frac{4}{n+1},
$$

and thus the function $|x|$ may be uniformly approximated by rational interpolation at the equidistant points with the rate at least $O(1 / n)$. This is in striking contrast to the classical result of Bernstein that the sequence of Lagrange interpolating polynomials to $|x|$ at equally spaced points in $[-1,1]$ diverges everywhere, except at zero and the end-points. (See for example, [3].) It should be mentioned that estimate (5) is a bit conservative, since it was proved by Werner in $[8]$ that the exact order of rational interpolation of $|x|$ at equidistant points is $O(1 / n \log n)$.

In the present paper we consider rational interpolation to $|x|$ corresponding to the set of the Chebyshev nodes which are known to be very efficient for polynomial interpolation. We show that the exact order of approximation of $|x|$ by rational interpolation at the Chebyshev nodes is also $O(1 / n \log n)$. Thus, in contrast to the polynomial case, for rational interpolation of $|x|$ the Chebyshev nodes are not better than the equidistant ones.

Finally we would like to mention that the method of our proof is rather general and may be applied to other specific sets of interpolation points. 


\section{Results}

Consider the case of the Chebyshev nodes, namely let

$$
X=T:=\left\{x_{k}\right\}=\sin ((2 k-1) \pi /(4 n)), \quad k=1,2, \ldots, n
$$

be the roots of the Chebyshev polynomial $T_{2 n}(x)$ of degree $2 n$, lying in $(0,1)$. Then, as can be easily verified,

$$
p(x) p(-x)=\frac{(-1)^{n} T_{2 n}(x)}{2^{2 n-1}},
$$

and therefore we have

$$
h_{n}(T ; x)=\frac{(-1)^{n} T_{2 n}(x)}{2^{2 n-1} p^{2}(x)} .
$$

Since $|x|$ and $r_{n}(X ; x)$ are even functions in $[-1,1]$ we can restrict ourselves to $x \in$ $[0,1]$. The following estimate holds:

LEMma 2.1.

$$
\left|h_{n}(T ; x)\right| \leqslant \frac{1}{4 n}, \quad x \in\left[x_{1}, 1\right] .
$$

Proof: Since $\left|T_{n}(x)\right| \leqslant 1$ for $x \in[-1,1]$, it follows from (6) that for $x \geqslant x_{1}$

$$
\left|h_{n}(T ; x)\right| \leqslant \frac{1}{2^{2 n-1} p^{2}(x)}=\frac{1}{2^{2 n-1} \prod_{k=1}^{n}\left(x+x_{k}\right)^{2}} \leqslant \frac{1}{2^{2 n-1} \prod_{k=1}^{n}\left(x_{1}+x_{k}\right)^{2}} .
$$

Let $B_{n}:=\prod_{k=1}^{n}\left(x_{1}+x_{k}\right)$. An easy computation reveals:

$$
\begin{aligned}
B_{n} & =\prod_{k=1}^{n}\left[\sin \frac{\pi}{4 n}+\sin \frac{(2 k-1) \pi}{4 n}\right]=\prod_{k=1}^{n} 2 \sin \frac{k \pi}{4 n} \prod_{k=2}^{n} \cos \frac{(k-1) \pi}{4 n} \\
& =\sqrt{2} \prod_{k=1}^{n-1} 2 \sin \frac{k \pi}{4 n} \cos \frac{k \pi}{4 n}=\frac{\sqrt{2}}{2} \prod_{k=1}^{2 n-1} \sin \frac{k \pi}{2 n} .
\end{aligned}
$$

By using the well-known identity (see for example, formula (1.392) in [4])

$$
\prod_{k=1}^{2 n-1} \sin \frac{k \pi}{2 n}=\frac{n}{2^{2 n-2}}
$$

we find

$$
B_{n}^{2}=\frac{n}{2^{2 n-3}}
$$

Combining (8) and (9) completes the proof of the lemma.

Now we are in a position to prove the following estimate for the approximation error: 
THEOREM 2.2 .

$$
\left|e_{n}(T ; x)\right| \leqslant \begin{cases}\frac{C_{1}}{n \log n}, & |x| \in\left[0, \sin \frac{\pi}{4 n}\right], \\ \frac{C_{2} \log n}{n^{2}}, & |x| \in\left[\sin \frac{\pi}{4 n}, \frac{\pi \log n}{n}\right], \\ \frac{C_{3}}{n^{2}}, & |x| \in\left[\frac{\pi \log n}{n}, 1\right] .\end{cases}
$$

Proof: As before we can restrict our analysis to $x \geqslant 0$. Consider first the case $x \in\left[0, x_{1}\right]=[0, \sin (\pi /(4 n))]$. In order to apply (4), we have to estimate the following sum:

$$
\begin{aligned}
A_{n}(T):=\sum_{k=1}^{n} x_{k}^{-1} & =\sum_{k=1}^{n} \frac{1}{\sin ((2 k-1) \pi / 4 n)}=\frac{1}{2} \sum_{k=1}^{2 n} \frac{1}{\sin ((2 k-1) \pi / 4 n)} \\
& =n\left[\frac{1}{2 n} \sum_{k=1}^{2 n} \frac{1}{\sin ((2 k-1) \pi / 4 n)}\right]=n \Lambda_{2 n-1}(T) .
\end{aligned}
$$

Here $\Lambda_{2 n-1}(T)$ is the Lebesgue constant for polynomial interpolation corresponding to the set of the Chebyshev nodes, for which the following two-sided inequality holds (see for example, $[\mathbf{6}])$ :

$$
a_{0}+\frac{2}{\pi} \log 2 n<\Lambda_{2 n-1}(T) \leqslant 1+\frac{2}{\pi} \log 2 n,
$$

where

$$
a_{0}=\frac{2}{\pi}\left(\gamma+\log \frac{8}{\pi}\right)=0.9625 \ldots, \quad \gamma \text { being Euler's constant. }
$$

Thus

$$
\left|e_{n}(T ; x)\right| \leqslant \frac{1}{A_{n}(T)}<\frac{1}{n\left(a_{0}+(2 / \pi) \log 2 n\right)} \leqslant \frac{C_{1}}{n \log n}
$$

Further consider the case $x>x_{1}$. Note first that in view of the lemma

$$
\left|e_{n}(T ; x)\right| \leqslant \frac{8}{3}\left|h_{n}(T ; x)\right|, \quad x \in\left[x_{1}, 1\right]
$$

and therefore we can restrict ourselves to finding an upper bound for $\left|h_{n}(T ; x)\right|$. To this end we apply (2) and take into account that for the Chebyshev nodes

$$
S_{1}(T)=\sum_{k=1}^{n} \sin (2 k-1) \frac{\pi}{4 n}=\sum_{k=1}^{n} \cos (2 k-1) \frac{\pi}{4 n}=\frac{1}{2 \sin (\pi / 4 n)} \geqslant \frac{2 n}{\pi} .
$$

In (13) we have used the well-known formula

$$
\sum_{k=1}^{n} \cos (2 k-1) t=\frac{\sin 2 n t}{2 \sin t}
$$


Thus we obtain

$$
\left|h_{n}(T ; x)\right| \leqslant e^{-(2 n x / \pi)}, \quad x \in[0,1] .
$$

Now we can require

$$
e^{-(2 n x / \pi)}<\frac{1}{n^{2}}=e^{-2 \log n}
$$

which will be satisfied assuming

$$
x>\frac{\pi \log n}{n}
$$

It remains to consider the intermediate interval $x \in[\sin (\pi / 4 n),(\pi \log n) / n]$. But in this case it follows from (1), in view of the lemma, that

$$
\left|e_{n}(T ; x)\right| \leqslant \frac{\left|2 x h_{n}(T ; x)\right|}{1-\left|h_{n}(T ; x)\right|} \leqslant\left(\frac{2 \pi \log n}{n}\right)\left(\frac{1}{4 n}\right)\left(\frac{4}{3}\right)=\frac{2 \pi \log n}{3 n^{2}}
$$

This completes the proof of the theorem.

Corollary 2.3. For any $x \in[-1,1]$ the following estimate holds:

$$
\left|e_{n}(T ; x)\right| \leqslant \frac{C}{n \log n}
$$

Finally we show that the estimate (14) is sharp, namely, the following result holds:

Theorem 2.4. Let $x^{\star}=1 /(n \log n)$. Then

$$
\left|e_{n}\left(T ; x^{\star}\right)\right| \geqslant \frac{C}{n \log n}, \quad n \geqslant n_{0} .
$$

Proof: Note first that for $n>3, x^{\star} \in\left[0, x_{1}\right]$ and since in this interval $0 \leqslant$ $h_{n}(T ; x) \leqslant 1$, we can write:

$$
n \log n\left|e_{n}\left(T ; x^{\star}\right)\right|=\frac{e_{n}\left(T ; x^{\star}\right)}{x^{\star}}=\frac{2 h_{n}\left(T ; x^{\star}\right)}{1+h_{n}\left(T ; x^{\star}\right)} \geqslant h_{n}\left(T ; x^{\star}\right) .
$$

Thus in order to prove (15) we have to show that the sequence $\left\{F_{n}\right\}_{n=1}^{\infty}$ defined by

$$
F_{n}:=\frac{1}{h_{n}\left(T ; x^{\star}\right)}=\frac{p^{2}\left(x^{\star}\right) 2^{2 n-1}}{(-1)^{n} T_{2 n}\left(x^{\star}\right)}
$$

is bounded. To this end note that

$$
\lim _{n \rightarrow \infty}\left[(-1)^{n} T_{2 n}\left(x^{\star}\right)\right]=1
$$


and therefore it suffices to consider the behaviour of the numerator of (16). Let

$$
P_{n}:=p^{2}\left(x^{\star}\right)=\prod_{k=1}^{n}\left(x^{\star}+x_{k}\right)^{2}=\prod_{k=1}^{n} x_{k}^{2} \prod_{k=1}^{n}\left(1+\frac{x^{\star}}{x_{k}}\right)^{2}:=Q_{n} * R_{n} .
$$

Then for the first factor we have

$$
Q_{n}=\prod_{k=1}^{n} \sin ^{2} \frac{(2 k-1) \pi}{4 n}=\prod_{k=1}^{2 n} \sin \frac{(2 k-1) \pi}{4 n}=\frac{1}{2^{2 n-1}},
$$

where the last equality is a well-known formula (see for example, formula 1.392(2) from $[4])$.

Thus it remains to verify that the sequence $\left\{R_{n}\right\}_{n=1}^{\infty}$ is bounded. Taking into account (11) and (12), we obtain

$$
\begin{aligned}
\log R_{n} & =2 \sum_{k=1}^{n} \log \left(1+\frac{x^{\star}}{x_{k}}\right) \leqslant 2 x^{\star} \sum_{k=1}^{n} \frac{1}{x_{k}}=2 x^{\star} A_{n}(T) \\
& =2 x^{\star} n \Lambda_{2 n-1}(T) \leqslant \frac{2}{\log n}\left(1+\frac{2}{\pi} \log 2 n\right) \leqslant C,
\end{aligned}
$$

and the result follows.

\section{REFERENCES}

[1] S. Bernstein, 'Sur la meilleure approximation de $|x|$ par des polynômes de degrés donnés', Acta Math. 37 (1913), 1-57.

[2] L. Brutman and E. Passow, 'On rational interpolation to $|x|$ ', Constr. Approx. (to appear).

[3] G.J. Byrne, T.M. Mills and S.J. Smith, 'On Lagrange's interpolation with equidistant nodes', Bull. Austral. Math. Soc. 42 (1990), 81-89.

[4] I.S. Gradsteyn and I.M. Ryzhik, Table of integrals, series and products (Academic Press, New York, 1980).

[5] D. Newman, 'Rational approximation to $|x|$ ', Michigan Math. J. 11 (1964), 11-14.

[6] T.J. Rivlin, Chebyshev polynomials, (2nd ed.) (Wiley, New York, 1990).

[7] H. Stahl, 'Best uniform rational approximation of $|x|$ on $[-1,1]$ ', Mat. Sb. 183 (1992), 85-118.

[8] H. Werner, 'Rationale Interpolation von $|x|$ in äquidistanten Punkten', Math. Z. 180 (1982), 85-118.

Department of Mathematics and

Computer Science

University of Haifa

Haifa 31905

Israel

e-mail: lev@mathcs.haifa.ac.il
Department of Mathematics

Temple University

Philadelphia PA 19122

United States of America

e-mail: passow@euclid.math.temple.edu 